\title{
Testing the backbone of the healthcare system: a prospective serological-epidemiological cohort study of healthcare workers in rural Germany.
}

Stephanie Hoffmann ( $\nabla$ stephanie.hoffmann@b-tu.de )

Brandenburg University of Technology Cottbus-Senftenberg Jacob Spallek

Brandenburg University of Technology Cottbus-Senftenberg

Heinz-Detlef Gremmels

Klinikum Niederlausitz GmbH

Juliane Schiebel

Klinikum Niederlausitz GmbH

Frank Hufert

Medical University Brandenburg Theodor Fontane

\section{Short Report}

Keywords: SARS-CoV-2, healthcare workers, rurality, virus prevalence

Posted Date: September 29th, 2020

DOI: https://doi.org/10.21203/rs.3.rs-84703/v1

License: (9) This work is licensed under a Creative Commons Attribution 4.0 International License. Read Full License 


\section{Testing the backbone of the healthcare system: a prospective serological- epidemiological cohort study of healthcare workers in rural Germany.}

Stephanie Hoffmann ${ }^{1 *}$, Juliane Schiebel ${ }^{2}$, Frank Hufert ${ }^{3}$, Heinz-Detlef Gremmels ${ }^{2}$, Jacob Spallek $^{1}$

${ }^{1}$ Department of Public Health, Institute of Health, Brandenburg University of Technology Cottbus-Senftenberg, Germany

${ }^{2}$ Institute for Clinical Chemistry, Laboratory Diagnostics and Microbiology, Klinikum Niederlausitz GmbH, Senftenberg, Germany

${ }^{3}$ Department of Microbiology and Virology, Medical University Brandenburg Theodor Fontane, Neuruppin, Germany

Correspondence to:

Stephanie Hoffmann

Department of Public Health

Brandenburg University of Technology Cottbus-Senftenberg

Universitätsplatz 1

01968 Senftenberg, Germany

Email: Stephanie.Hoffmann@b-tu.de 


\section{ABSTRACT}

Healthcare workers are of a high priority in SARS-CoV-2 pandemic and epidemiological control strategies due to their relevance for the healthcare system and high exposure levels that might result in symptomatic and asymptomatic courses. Aim of this study is to assess the prevalence of SARS-CoV-2 infections in a cohort of 171 healthcare workers in a standard care hospital in a rural German area. The outcomes are monthly measures of antibody prevalence over a 6-month period. Using univariate statistics, baseline characteristics will be analysed considering risk groups, exposure history, test frequencies, symptoms, and antibody distribution.

At T0 tests for qualitative detection of antibodies (Ig) against SARS-CoV-2 revealed a seroprevalence of $1.3 \%$.

The data contribute to the understanding of the virus spread in a particularly exposed population group and will be relevant for management of the pandemic. Longitudinal analyses will reveal the progression of seroprevalence of antibodies and immunity over the time.

\section{INTRODUCTION}

As of August 2020, the coronavirus (SARS-CoV-2) was pandemic ${ }^{1}$, and has affected 242,381 people in Germany and 3,870 of them in Brandenburg ${ }^{2}$ with COVID-19. Implemented population-related hygiene activities in Germany support overall good protection against spread of virus ${ }^{3}$, leading to falling incidences in federal states, such as Brandenburg ${ }^{4}$. Nevertheless, differences in exposure risks depend on, amongst others, contextual circumstances, such as working conditions ${ }^{5,6}$. Therefore, healthcare workers $(\mathrm{HCW})$ are an essential target population in infection control of SARS-CoV- $2^{7}$, because of their higher probability of exposure to sources of COVID-195,8,9 and transmission of infectious diseases ${ }^{2}$. Since March 2020 an increasing amount of cases among HCW has been documented in Anglo-Saxon countries $^{9,10}$, and nationally, that leaded to a minimum value of $6.3 \%$ of all prevalent cases in Germany occupied in healthcare facilities (e.g. hospitals, outpatient clinics 
and practices, dialysis clinics or outpatient nursing services) at 31th August 2020. In 23 of these cases, COVID-19 became fatal' .

Besides job-related variations in exposure risks and in risks of reporting positive tests for COVID-19 ${ }^{10}$, regional differences have been documented with regard to both federal states and districts. Geographical distribution of laboratory-confirmed COVID-19 cases in the past seven days from 31th August 2020 per 100,000 population varies from 62,6 in federal state of Mecklenburg-Western Pomerania to 437,6 in federal state of Bavaria (federal state of Brandenburg: 154,1)2. In health departments in Brandenburg districts diverse 7-day incidence per 100,000 population could be calculated, with no new cases especially in the district of this study, Oberspreewald-Lausitz, at 2th September $2020^{11}$. As a rural sparsely populated area, Oberspreewald-Lausitz reports comparatively low confirmed cases (July: 58, August: $69^{11}$ ).

However, while there is evidence about regional and job-related differences in risk of infection, little is known so far about the prevalence of serum antibodies against SARS-CoV-2 in a population of HCW in standard care hospitals in rural German districts. It is crucial to generate evidence about degree of infection spread by serological examinations of HCW to develop additionally protection strategies and to ensure adequate healthcare provision, as an infection with SARS-CoV-2 leads to the production of different classes of antibodies directed against the $\mathrm{S}$ or $\mathrm{N}$ protein, usually detectable in the second week after onset of symptoms ${ }^{12}$. Therefore a prospective cohort study was established to analyse symptomatic and asymptomatic disease courses among $\mathrm{HCW}$ and related antibody prevalence aiming at developing loping ensuring adequate healthcare

\section{METHODS}

Following a longitudinal approach, HCW $(n=171)$ who agreed on being examined monthly over a 6-month period were recruited in July 2020 in a standard care hospital in Oberspreewald-Lausitz. The representative sample consist of staff of medical, nursing, therapeutical, laboratory, as well as radiology and service/ administration sector. Due to 
different risk exposure levels, participants are clustered in four groups according to Occupational Risk Pyramid for COVID-195 (see Figure 1).

Epidemiologic and demographic data (e.g. sex, age, comorbidities), exposure history, as well as data on stress related to the corona pandemic are collected using standardised instruments in a quantitative questionnaire.

To identify SARS-CoV-2 antibodies blood samples are collected and processed on two consecutive days. Serum is tested by an immunological test for the qualitative detection of antibodies (total lg) against SARS-CoV-2. The assay uses a recombinant protein that is the nucleocapsid (N) antigen of SARS-CoV-2. In short, this technique is based on a double antigen sandwich principle in which the test sample, the biotinylated SARS-CoV-2 specific recombinant antigen and the ruthenium labeled SARS-CoV-2 specific recombinant antigen are incubated together. After addition of streptavidin-coated micro particles, the complex is bound to a solid phase by the interaction between biotin and streptavidin and transferred to a measuring cell. The micro particles are magnetically fixed, unbound material is removed and after applying a voltage, the chemiluminescence emission is measured with a photomultiplier. The result is known as a cut-off index $(\mathrm{COI})$, whereby a $\mathrm{COI} \geq 1.00$ is interpreted as reactive, i.e. positive for SARS-CoV-2 antibodies. For this test the sensitivity of the detection of SARS-CoV-2 antibodies 14 days after a positive polymerase chain reaction (PCR) test is $99.5 \%(n=185)$ and the specificity is $99.8 \%(n=10453)$. To exclude an acute infection in case of a positive antibody test, a real-time RT-PCR ${ }^{13}$ (Xpert Xpress SARS-CoV-2, Cepheid) with a nasopharyngeal swab of the affected persons was performed on the same day of blood collection.

Additionally, contextual data including confirmed COVID-19 cases overall in the district Oberspreewald-Lausitz and particularly cared for in the examined hospital are compiled. Further data about national and federal COVID-19 management (e.g. governmental regulations, contact restrictions, distance requirements, and protective clothing), events (e.g. local COVID-19 outbreaks) as well as weather data (e.g. daily sunshine duration, daily mean of vapor-pressure, and daily mean of temperature) are recoded. 
The primary outcomes of the study are measures of antibody prevalence in terms of COI among HCW. Data collection will continue until December 2020. Using univariate statistics (e.g. frequencies), baseline characteristics will be analysed considering risk groups, contact history, test frequencies, acute symptoms, and antibody distribution. In a longitudinal approach multivariate analysis (e.g. regression analysis) regarding the influence of individual (e.g. contact history, stress) and contextual exposures are planned to gain deeper understanding of virus spreading and immunity development among high risk population groups.

All methods are carried out in accordance with relevant guidelines and regulations. Blood samples are obtained by trained personnel. A standardized protocol to analyze human blood serum by an enzyme-linked immunosorbent assay is applied ${ }^{14}$. Since parts of the body can be qualified as personal data, the blood samples are refrigerated kept in a biobank applying EU General Data Protection Regulation and German Federal Data Protection Act. Laboratory testings are based on biosafety and testing guidelines published by the World Health Organization ${ }^{15,16}$ and recommendations issued by the German Robert Koch Institute ${ }^{17}$. Epidemiological and stress-related data in accompanying questionnaire are collected and analysed with regard to Guidelines and recommendations for ensuring Good Epidemiological Practice $^{18}$.

\section{RESULTS}

At baseline (T0) all required data from questionnaire and blood samples are available in $n=156$ cases (female $86.5 \%)$, which are grouped as in very high risk $(n=30)$, high risk $(n=54)$, medium risk $(n=31)$ and lower risk $(n=41)$ (see table 1$)$. While very high or high risk group include mostly nurses, physicians and therapists, radiology or lab assistants belong to medium risk population and service and administration staff are grouped in the lowest risk group.

Since May 2020 overall six COVID-19 patients have been cared for in the hospital. Contact with confirmed COVID-19 cases in the last four weeks is most frequently in very high risk staff $(20.0 \%)$. Among these contacts, all are job-related in this risk group. Compared to that, exposure to COVID-19 is not entirely job-related in high risk group (71.4\%) or medium 
risk group (80.0\%). Among other disease symptoms within the last four weeks, such as sore throat, rhinitis and gastrointestinal distress, fatigue is common across all risk groups. Within the last four weeks overall nine SARS-CoV-2 tests have been performed, with no positive results of an acute infection with SARS-CoV-2. Of a total of $n=156$ serum samples analyzed, two cases show reactive antibodies (1.3\%), one of them previously undiscovered. While the $\mathrm{COI}$ of 60.12 occurs in the blood sample of a participant belonging to high risk group, the $\mathrm{COI}$ of 97.31 has shown in very high risk population. The COI of all negative blood samples was between 0.05 and 0.23 . The PCR for SARS-CoV-2 was negative in both antibody-positive participants.

\section{DISCUSSION}

Due to a lack of comprehensive data, the knowledge about prevalence of SARS-CoV2 infections in different population groups of people is still scarce. The findings of a low antibody prevalence of $1.3 \%$ are in line with existing knowledge gained in other seroepidemiological studies according to the spread of SARS-CoV-2 in population groups in Germany. For example, in medical staff of a maximum care hospital in Fulda a low seroprevalence of $\lg$ antibodies about $1.0 \%$ and of $\lg A$ antibodies about $4.4 \%$ was detected $^{19}$. Also among public service employees in Bremen the estimated seroprevalence of $2.1 \% \lg G$ antibodies is to be assessed quiet low ${ }^{20}$. Compared to seroprevalence in blood donors of $0.91 \%$ of IgG antibodies against SARS-CoV- $2^{21}$ the prevalence is slightly higher. The sensitivity and specificity of the used test are in line with developed antibody tests ${ }^{13}$. But the level of the $\mathrm{COI}$ is not indicative for the total amount of antibodies in the sample, nor does it evaluate the effectiveness of the antibodies. In further research this should be investigated using a neutralization test. In this test, cell culture assays are used to determine whether the antibodies formed against SARS-CoV-2 are able to neutralize the virus and thus protect the cells from infection. First studies indicate that these effective antibodies are detectable towards the end of the 2 nd week after the onset of symptoms ${ }^{22-25}$. Investigations into protective titers 
and stability of the immunity are not yet known, but single cases with ineffective antibodies have already been described ${ }^{26,27}$.

HCW play an important role during a pandemic and monitoring of occurrence and spread of the virus in this particularly exposed group should be an essential part of pandemic control measures. The data of this regional cohort contribute to the epidemiological understanding of the virus spread in this particularly exposed population group and will be relevant for further management of the pandemic. Longitudinal analyses of the cohort will reveal the progression of seroprevalence of antibodies and antibody titer against SARS-CoV2 in HCW over the time.

\section{ETHICS DECLARATIONS}

As primary data and blood samples were collected, ethical approval for complete study was given by the ethics committee of Brandenburg University of Technology CottbusSenftenberg (EK2020-8). Written informed consent for participation in this study was obtained from all participants.

\section{AUTHOR CONTRIBUTIONS}

JSp, SH, HDG and JSch designed the study. JSp and HDG are the PIs of study. JSch and HDG coordinate the recruitment and data collection in the hospital, SH and JS coordinate the data management and analysis. FH contributes to microbiological and data analysis. All authors participated in all aspects of data analysis. SH wrote the first draft of the manuscript, which was then revised by all authors. All authors have read and approved the final version.

\section{COMPETING INTERESTS STATEMENT}

No financial support was provided to this study and related articles. All authors report no conflicts of interest to this article. 


\section{DATA AVAILABILITY}

Large parts of the dataset generated and analysed during this study are not publicly available due to German data protection laws. Data and questionnaires will be made accessible to researchers based on an access proposal in line with the German data protection regulations. 


\section{REFERENCES}

1. World Health Organization. WHO Coronavirus Disease (COVID-19) Dashboard. Available at https://covid19.who.int/ (2020).

2. Robert Koch-Institut. Coronavirus Disease 2019 (COVID-19) Daily Situation Report of the Robert Koch Institute. 31/08/2020. Available at https://www.rki.de/DE/Content/InfAZ/N/Neuartiges_Coronavirus/Situationsberichte/Archiv _August.html.

3. Ministerium für Soziales, Gesundheit, Integration und Verbraucherschutz des Landes Brandenburg (MSGIV). Verordnung über den Umgang mit dem SARS-CoV-2-Virus und COVID-19 in Brandenburg. Gesetz- und Verordnungsblatt für das Land Brandenburg Teil II - Verordnungen.

4. Ministerium für Soziales, Gesundheit, Integration und Verbraucherschutz des Landes Brandenburg (MSGIV). Bestätigte COVID-19-Fälle in Brandenburg. Available at https://msgiv.brandenburg.de/msgiv/de/presse/pressemitteilungen/detail/ 08-07-2020corona-faelle-stand-08072020.

5. U.S. Department of Labor Occupational Safety and Health Administration. Guidance on Preparing Workplaces for COVID-19. Available at www.osha.gov.

6. Angerer, P., Kaifie-Pechmann, A. \& Tautz, A. Beschäftigte mit erhöhtem Krankheitsrisiko. Umgang mit Risikogruppen für schwere Krankheitsverläufe an Arbeitsplätzen oder in Tätigkeiten mit erhöhtem COVID-19 Infektionsrisiko. Available at https://www.publichealth-covid19.de/ (2020).

7. Robert Koch-Institut. Management von COVID-19 Ausbrüchen im Gesundheitswesen. Available at https://www.rki.de/DE/Content/InfAZ/N/Neuartiges_Coronavirus/Management_Ausbruch_ Gesundheitswesen.html.

8. Chirico, F., Nucera, G. \& Magnavita, N. COVID-19: Protecting Healthcare Workers is a priority. Infection control and hospital epidemiology, 1-4 (2020). 
9. Centers for Disease Control and Prevention. Characteristics of Health Care Personnel with COVID-19 - United States, February 12-April 9, 2020. MMWR Morb Mortal Wkly Rep 2020. MMWR. Morbidity and mortality weekly report 69, 477-481 (2020).

10. Nguyen, L.H. et al. Risk of COVID-19 among front-line health-care workers and the general community: a prospective cohort study. The Lancet Public Health 5, e475-e483 (2020).

11. Robert Koch-Institut. COVID-19-Dashboard. Landkreis Oberspreewald-Lausitz. Available at https://experience.arcgis.com/experience/478220a4c454480e823b17327b2bf1d4/page/p age_1/.

12. Kellam, P. \& Barclay, W. The dynamics of humoral immune responses following SARSCoV-2 infection and the potential for reinfection. The Journal of general virology 101, 791-797 (2020).

13. Horvath, K., Semlitsch, T., Jeitler, K., Krause, R. \& Siebenhofer, A. Antikörpertests bei COVID-19 - Was uns die Ergebnisse sagen. Zeitschrift fur Evidenz, Fortbildung und Qualitat im Gesundheitswesen 153, 54-59 (2020).

14. Guan, M. et al. Recombinant protein-based enzyme-linked immunosorbent assay and immunochromatographic tests for detection of immunoglobulin $\mathrm{G}$ antibodies to severe acute respiratory syndrome (SARS) coronavirus in SARS patients. Clinical and diagnostic laboratory immunology 11, 287-291 (2004).

15. World Health Organization. Laboratory testing of 2019 novel coronavirus (2019-nCoV) in suspected human cases: interim guidance. (2020).

16. World Health Organization. Laboratory biosafety guidance related to coronavirus disease (COVID-19). Interim guidance. (2020).

17. Robert Koch Institut. Hinweise zur Testung von Patienten auf Infektion mit dem neuartigen Coronavirus SARS-CoV-2. Available at https://www.rki.de/DE/Content/InfAZ/N/Neuartiges_Coronavirus/Vorl_Testung_nCoV.html 
18. Hoffmann, W. et al. Guidelines and recommendations for ensuring Good Epidemiological Practice (GEP): a guideline developed by the German Society for Epidemiology. European journal of epidemiology 34, 301-317 (2019).

19. Kern, P.M., Müller, H.-H., Menzel, T. \& Weisser, H. Studie zur Immunität gegen SARSCoV-2. Der Klinikarzt 49, 268-273 (2020).

20. Neumann, J., Zeeb, H., Dotzauer, A. \& Janssen-Weets, O. Low anti-SARS-CoV-2 seroprevalence among public sector employees in Bremen. Zeitschrift für medizinische Prävention 08 (2020).

21. Fischer, B., Knabbe, C. \& Vollmer, T. SARS-CoV-2 IgG seroprevalence in blood donors located in three different federal states, Germany, March to June 2020. Europe's journal on infectious disease surveillance, epidemiology, prevention and control 25 (2020).

22. Wölfel, R. et al. Virological assessment of hospitalized patients with COVID-2019. Nature $581,465-469$ (2020).

23. Amanat, F. et al. A serological assay to detect SARS-CoV-2 seroconversion in humans. Nature medicine 26, 1033-1036 (2020).

24. Zhao, J. et al. Antibody responses to SARS-CoV-2 in patients of novel coronavirus disease 2019. Clinical infectious diseases : an official publication of the Infectious Diseases Society of America (2020).

25. Okba, N.M.A. et al. Severe Acute Respiratory Syndrome Coronavirus 2-Specific Antibody Responses in Coronavirus Disease Patients. Emerging infectious diseases 26, 14781488 (2020).

26. Wu, F. et al. Neutralizing antibody responses to SARS-CoV-2 in a COVID-19 recovered patient cohort and their implications (2020).

27. Payne, D.C. et al. SARS-CoV-2 Infections and Serologic Responses from a Sample of U.S. Navy Service Members - USS Theodore Roosevelt, April 2020. MMWR. Morbidity and mortality weekly report 69, 714-721 (2020). 


\section{FIGURE LEGENDS}

Figure 1. Clustering strategy by risk group

\begin{tabular}{|c|c|c|c|}
\hline very high risk & high risk & medium risk & lower risk \\
\hline $\begin{array}{l}\cdot \text { emergency room } \\
\text { - intensive care } \\
\text { medicine }\end{array}$ & $\begin{array}{l}\text { - cardiology } \\
\text { - geriatrics } \\
\text { - pediatrics }\end{array}$ & $\begin{array}{l}\text { - laboratory } \\
\text { - radiology }\end{array}$ & - administration \\
\hline
\end{tabular}




\section{TABLES}

Table 1. Participant characteristics ( $n=156)$, SARS-CoV-2 contacts, antibody distribution, test frequencies, acute symptoms

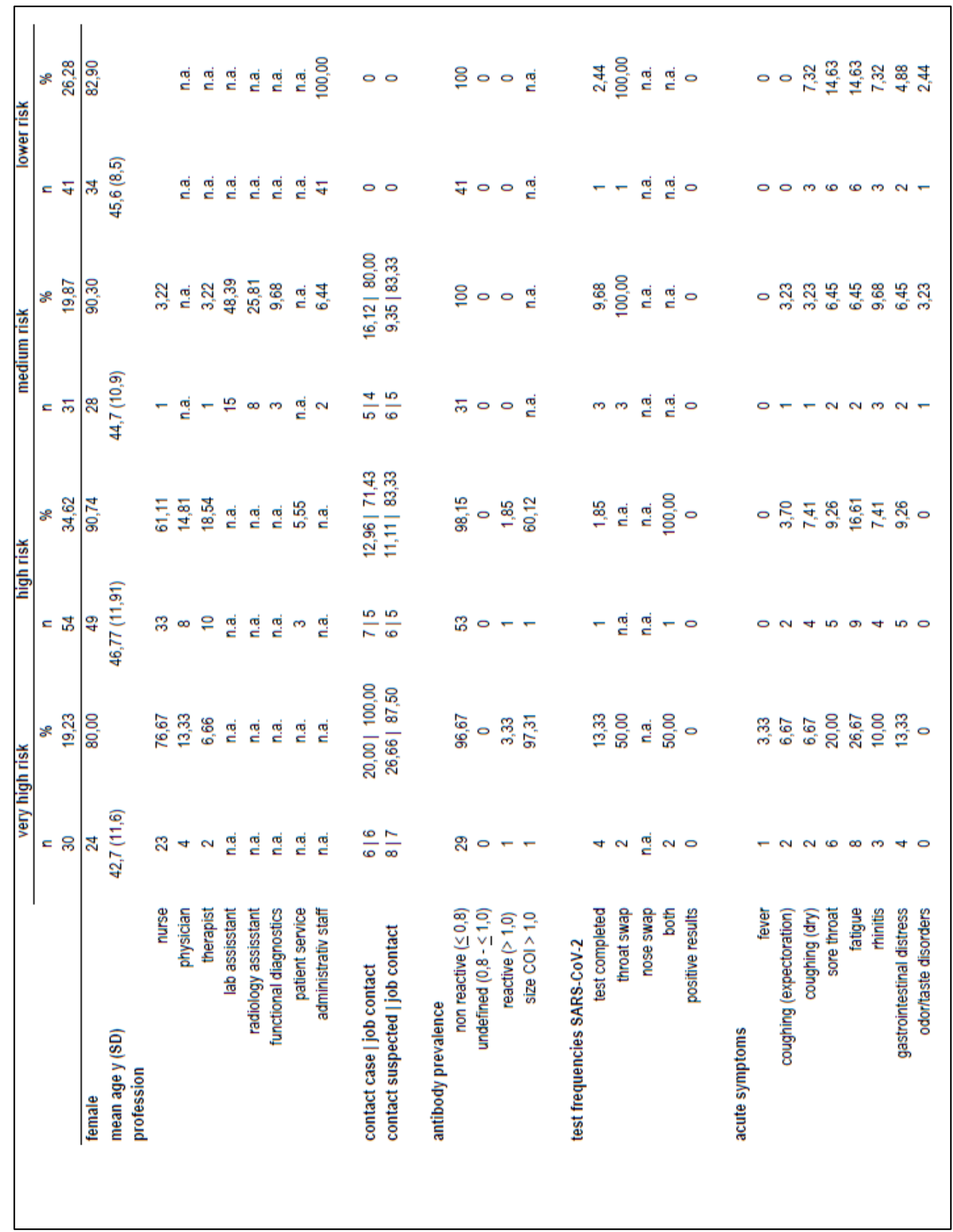


Figures

\begin{tabular}{|l|l|l|l|}
\hline \multicolumn{1}{|c|}{ very high risk } & \multicolumn{1}{c|}{ high risk } & \multicolumn{1}{c|}{ medium risk } & \multicolumn{1}{c|}{ lower risk } \\
\hline $\begin{array}{l}\text {-emergency room } \\
\text {-intensive care } \\
\text { medicine }\end{array}$ & $\begin{array}{l}\text {-cardiology } \\
\text {-geriatrics } \\
\text {-pediatrics }\end{array}$ & $\begin{array}{l}\text {-laboratory } \\
\text {-radiology }\end{array}$ & -administration \\
\hline
\end{tabular}

Figure 1

Clustering strategy by risk group 